\title{
Periodicities in microwave radio bursts of solar flares
}

\author{
Zongjun Ning ${ }^{1}$ and M. D. Ding ${ }^{2}$ \\ ${ }^{1}$ Purple Mountain Observatory, Nanjing, 210008, China \\ email: ningzongjun@hotmail.com \\ ${ }^{2}$ Department of Astronomy, Nanjing University, Nanjing 210093, China
}

\begin{abstract}
We analyze the periodicities for 294 solar flares observed by Nobeyama Radioheliograph over 6 discrete frequencies of 1, 2, 3.75, 9.4, 17 and $35 \mathrm{GHz}$ during the solar cycle 23 between 1998 and 2004. Using Fourier analysis, we pick up the shortest period $\left(T_{\mathrm{s}}\right)$ for each event at each frequency channel. We find that $T_{\mathrm{s}}$ varies in the range of $0.25-0.62 \mathrm{~s}$ over 6 frequency channels.
\end{abstract}

Keywords. Sun: flares, Sun: radio radiation

\section{Introduction}

Nonthermal electrons generated in solar flares are responsible for hard X-ray (HXR) and radio emissions. Sometimes, HXR and/or radio emissions show periodicities or quasiperiodicities in solar flares. Investigtion of them can reveal the nature of electron acceleration and energy release processes. Therefore, this topic attracted much attention in the past decades (e.g. Nakajima et al. 1983; Aschwanden et al.1998; Asai et al. 2001). In this paper, we use Fourier analysis to search for the possible periodicities in microwave radio bursts.

\section{Data Analysis Method}

We analyze the periodicities in microwave bursts for 294 solar flares during the solar cycle 23 between 1998 and 2004 using the Fourier analysis (e.g. Delache et al. 1985; Lou et al. 2003). We use the time derivative of the flux instead of the original time profiles in Fourier analysis. This method was used by Manganey \& Pick (1989) and Zhao et al. (1991) to detect the periodicities in type III bursts. As was mentioned by these authors, to use the derivative of the time profile to compute the power spectrum is equivalent to multiply the power spectrum of the original time profile by a local frequency and to diminish the relative importance of the lower frequencies. This does not affect the high frequency domain that we are interested in.

\section{Results}

Figure 1 gives the distributions of $T_{\mathrm{s}}$ for 294 events over 6 discrete channels. We fit each distribution with a Gaussian curve to determine the statistical mean value and its range. The former is defined as the center of the Gaussian curve, while the latter is regarded as the full width at half maximum. We find that $T_{\mathrm{s}}$ is in the range of $0.25-0.62 \mathrm{~s}$. The values of $T_{\mathrm{s}}$ decreases with increasing frequencies with a relationship $T_{s}=0.384-0.026 \ln f$, where $f$ is the frequency. 


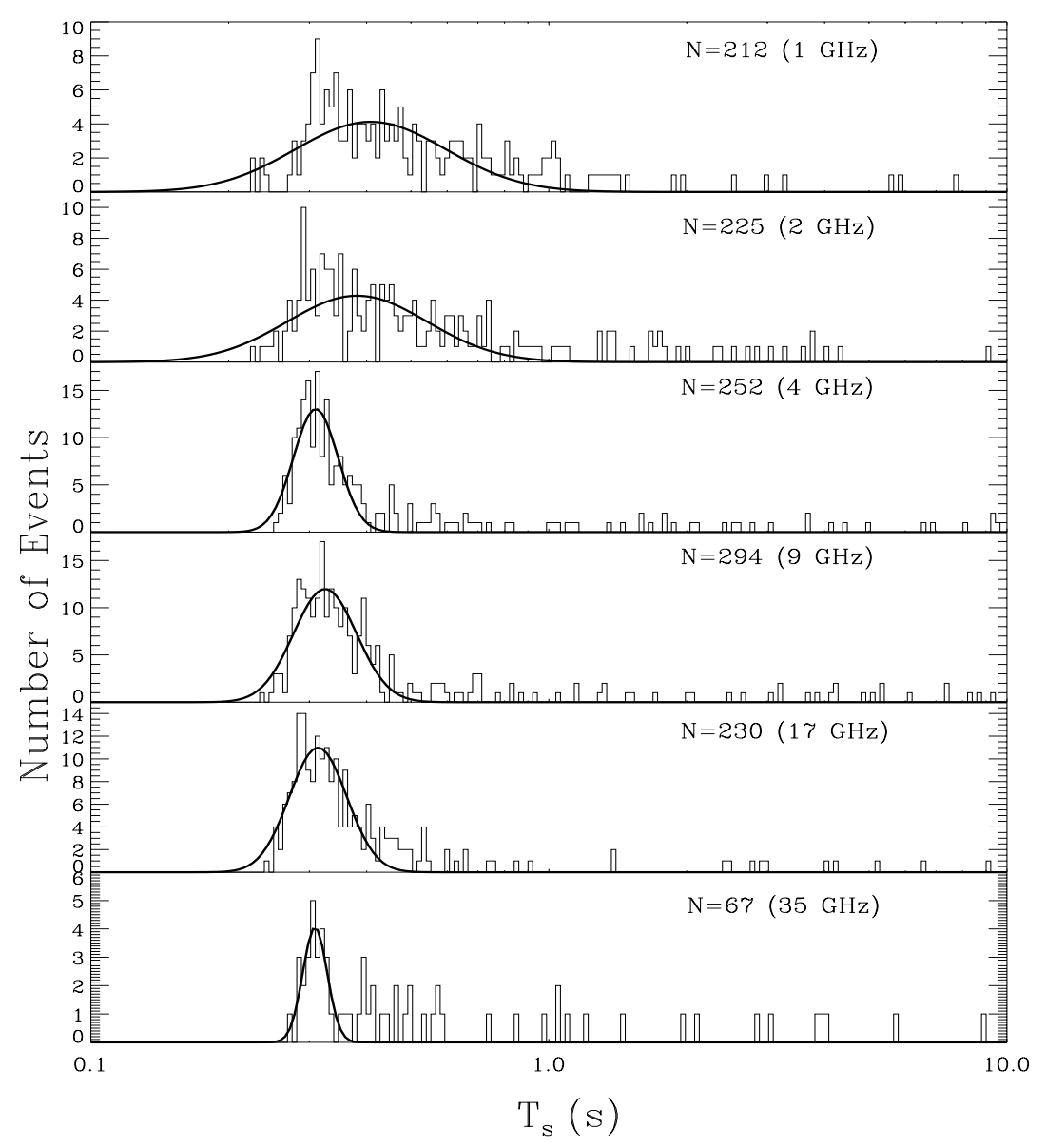

Figure 1. Histogram for the distribution of the shortest period, $T_{\mathrm{s}}$, over the $1,2,4,9,17$ and $35 \mathrm{GHz}$ wavelength channels respectively. The solid lines are Gaussian fitting for determination of the statistical mean value of $T_{\mathrm{s}}$ and its range.

\section{Acknowledgements}

This work was supported by NKBRSF under grant G20000784, by NSFC under grants 10025315, 10221001, 10279025, 10333030, and 10333040 .

\section{References}

Asai, A., Shimojo, M., Isobe, H., Morimoto, T., Yokoyama, T., Shibasaki, K., \& Nakajima, H. 2001, ApJ (Letters), 562, L103

Aschwanden, M. J., Kliem, B., Schwarz, U., Kurths, J., Dennis, B. R., \& Schwartz, R. A. 1998, ApJ, 505, 941

Delache, P., Laclare, F., \& Sadsaoud, H. 1985, Nature, 317, 416

Mangeney, A., \& Pick, M. 1989, Astro.Astrophy, 224, 242

Lou, Y.-Q., Wang, Y.-M., Fan, Z., Wang, S., \& Wang, J. X. 2003, Monthly Notice of the Royal Astronomical Society, 345, 809

Nakajima, H., Kosugi, T., Kai, K., \& Enome, S. 1983, Nature, 305, 292

Zhao, R.-Y., Mangeney, A., \& Pick, M. 1991, Astro.Astrophy., 241, 183 